risk with respect to other workers at comparable exposure levels are still object of debate. Overall, the topic of workers at particular risk exposed to EMF is an open question and has to be managed case by case using a combination of information sources: directive itself, exposure assessment in the workplace, technical standards, findings of workers' health surveillance, information acquired by the general practitioner or specialists having in care the worker, data from the manufacturer of the devices (e.g. technical sheets or instruction manuals), scientific literature etc.

\section{$1649 \mathrm{~d}$ WHAT HEALTH SURVEILLANCE OF EMF EXPOSED WORKERS?}

${ }^{1} \mathrm{~F}$ Gobba, ${ }^{2} \mathrm{~L}$ Korpinen. ${ }^{1}$ University of Modena and Reggio Emilia, Modena, Italy; ${ }^{2}$ Clinical Physiology and Neurophysiology Unit, The North Karelia Central Hospital, Joensuu, Finland

\subsection{6/oemed-2018-ICOHabstracts. 1205}

In the European Union (EU), the Directive 2013/35/EU has introduced the legal obligation of an 'appropriate health surveillance' (HS) for workers exposed to electromagnetic fields (EMF). Until now no agreement exists on the criteria, and on the contents, of such an HS. The EU Directive specifically refers to the protection from the risks associated with known direct biophysical and indirect short-term effects caused by EMF, while does not address to the suggested long-term effects since scientific evidence of a causal relationship is considered not adequate. Accordingly, at least in EU Countries specific objectives of HS are:

- the prevention of established effect, such as the stimulation of muscles, nerves or sensory organs (including temporary annoyance or effects on cognition) and limb currents, or any thermal effects;

- the health and safety of workers 'at particular risk', e.g. workers with active implanted medical devices (cardiac pacemakers, ICD, insulin pumps, etc.) or pregnant workers; nevertheless a comprehensive definition of the conditions inducing a 'particular risk', and of the safe thresholds, are still lacking.

It should be noted here that the exposure limits introduced by the Directive 2013/35/UE do not necessarily provide an adequate protection of such workers, e.g. interference problems with pacemakers may occur at lower levels. HS is therefore mainly aimed to evidence the occurrence of clinical symptoms possibly related to EMF and the existence of conditions possibly inducing particular risk, while specific laboratory test are not required, except on individual clinical basis. As a conclusion, at present time no agreement exists on the health surveillance of EMF exposed workers, and knowledge on some aspects, e.g. the conditions inducing particular risk and the possible thresholds, are still insufficient, and do not give an adequate support to the occupational physician to face the problem.

\section{MAGNETIC RESONANCE IMAGING (MRI) WORKERS: EMF EXPOSURE, OCCUPATIONAL RISK AND PREVENTION. AN UPDATE}

Fabriziomaria Gobba. University of Modena and Reggio Emilia, Modena, Italy

10.1136/oemed-2018-ICOHabstracts. 1206
Aim of special session Magnetic Resonance Imaging (MRI) is an important technology both for diagnostic and research purposes. MRI operators are exposed to high levels of electromagnetic fields (EMF), mainly static magnetic fields and lowfrequency time-varying magnetic fields (TvMF). Objective of this Special Session is an update of the results of research on the effects related to occupational EMF exposure in MRI operators, and on possible prevention.

Fabriziomaria Gobba ${ }^{1}$, Gian Marco Contessa ${ }^{2}$, Jolanta Karpowicz $^{3}$

${ }^{1}$ University of Modena and Reggio Emilia, Modena, Italy International Labour Office, Geneva, Switzerland

${ }^{2}$ Centro Ricerche Casaccia - ENEA, Rome, Italy

${ }^{3}$ Central Institute for Labour Protection - National Research Institute, Warszawa, Poland

\section{0a SUBJECTIVE SYMPTOMS IN MAGNETIC RESONANCE IMAGING OPERATORS: PRELIMINARY RESULTS OF AN ITALIAN STUDY}

${ }^{1} \mathrm{G}$ Zanotti, ${ }^{1} \mathrm{~A}$ Modenese, ${ }^{2} \mathrm{G}$ Bravo, ${ }^{3} \mathrm{G}$ Arcangeli, ${ }^{4} \mathrm{~V}$ Camisa, ${ }^{5} \mathrm{G}$ Corona, ${ }^{6} \mathrm{~S}$ Giglioli, ${ }^{7} \mathrm{G}$ Ligabue, ${ }^{8} \mathrm{R}$ Moccaldi, ${ }^{3} \mathrm{~N}$ Mucci, ${ }^{9} \mathrm{~L}$ Vimercati, ${ }^{4} \mathrm{~S}$ Zaffina, ${ }^{1} \mathrm{~F}$ Gobba. ${ }^{1}$ Dept. of Biomedical, Metabolic and Neural Sciences, University of Modena and Reggio Emilia, Modena, Italy; ${ }^{2}$ Dept. of Medicine, University of Udine, Italy; ${ }^{3}$ Dept. Experimental and Clinical Medicine, University of Florence; ${ }^{4}$ Occupational Medicine, Bambino Gesù Children's Hospital, IRCCS, Rome, Italy; ${ }^{5}$ Medico Competente Azienda Ospedaliero-Universitaria Policlinico di Modena; ${ }^{6}$ Medico Competente Azienda Unità Sanitaria Locale Siena; ${ }^{7}$ Radiologia 1 - Azienda Ospedaliero-Universitaria Policlinico di Modena; ${ }^{8}$ CNR-SPP Roma; ${ }^{9}$ Interdisciplinary Dept. of Medicine, Occupational Medicine 'B.Ramazzini', University of Bari

\subsection{6/oemed-2018-ICOHabstracts. 1207}

Introduction Magnetic Resonance Imaging (MRI) operators are exposed to significant levels of electromagnetic fields (EMF). In these workers an elevated prevalence of variuos subjective symptoms has been reported. Even if most symptoms are nonspecific, in some recent studies a group of 5 more specific symptoms, defined 'core symptoms', was proposed: vertigo, nausea, head ringing, magnetophosphenes and metallic taste. Our aim was to investigate the prevalence of subiective symptoms, including 'core symptoms', in Italian MRI operators, in order to evaluate the association with exposure.

Methods A sample of 152 MRI operators working in 6 different hospitals in Italy was collected. No measurments were available, so for each participant exposure was estimated based on scanner type $(<1 \mathrm{~T}, 1.5 \mathrm{~T}-<3 \mathrm{~T}$ and $\geq 3 \mathrm{~T}$ respectively) and on the total number of MRI procedures followed. In all operators an ad hoc questionnaire including relevant sociodemographic and occupational data, and the occurrence of subjective symptoms based our previous experience, was collected. The influence of stress was also evaluated. The relation between occupational exposure and symptoms was studied using multivariate analysis.

Result The multivariate analysis shows a significant correlation between exposure level and the total number of investigated symptoms. The results were confirmed considering the specific subset of 'core symptoms' based on previous literature data. In the multivariate model, the weight of the other covariate factors was not significant. The overall results were substantially confirmed considering the effect of work stress.

Discussion and conclusions In the observed group of MRI operators the total number of subjective symptoms was associated with EMF exposure; the subset of 'core symptoms' also proved significantly dose-related with exposure. The symptoms 